\title{
Diffusion on Fractal Substrates
}

\section{Raf Dekeyser, ${ }^{1}$ Amos Maritan ${ }^{2}$ and Attilio L. Stella ${ }^{2 \star}$}

1 Instituut voor Theoretische Fysica

Katholieke Universiteit Leuven, B-3030 Leuven, Belgium

2 Dipartimento di Fisica e Sezione I.N.F.N., Universitá di Padova, I-35131 Padova, Italy

\section{Introduction}

The study of static (geometric) properties of fractals has by now been widely accepted as the proper tool for describing physical objects that exhibit some kind of self-similarity [1, 2]. Lately, a lot of attention has also been devoted to the dynamical properties of fractals. This was triggered by some experiments in which the energy density of the vibrational states of some supposedly fractal polymers played an important role. These excitations were called "fractons" by Alexander and Orbach [3], who also pointed out the relation between these fractons and other dynamical properties like diffusion and resistivity [4].

In this contribution we first briefly review the basic concepts in this domain and the relation between the various dynamical phenomena. Thereafter, we discuss two different deterministic fractal structures for which the dynamical properties can be studied exactly by an iterative calculation. The first model, inspired by numerical studies of diffusion on random walk substrates, will give rise to a power-law dynamical behaviour, with fractal dynamical exponents that depend in a continuous way on a free parameter of the model. The second model leads to a logarithmic time-dependence of the diffusion, instead of a power-law behaviour.

Both examples constitute, in a sense, frontier applications of two important paradigms, which, from the start, played a very important role in the field. The first one is the use of deterministic hierarchical or fractal lattices to model, at least qualitatively, the complicated random self-similarity found in nature [5]. The simplifications inherent in such modelling make the application of renormalization group (RG) methods, our second paradigm, natural and feasible. We speak here of frontier applications in the sense that the model calculations that we deal with aim at an explanation of quite complicated issues, whose control - even numerically - is sometimes at the border of our present computational possibilities. In one case, the difficulty of the problem leads us to the necessity of reconsidering the standard strategy for extracting information on critical behaviour from the RG flow.

\footnotetext{
* With acknowledgement for financial support by the I.N.F.M.
} 


\section{Fractal Diffusion and Other Dynamical Properties}

Let us concentrate on a $d$-dimensional lattice, on which a fractal structure with Hausdorff-dimension $D$ is defined (e.g., by deleting from the lattice some specific sites and bonds). The diffusion equation describes the time evolution of the probability $P_{i}(t)$ to find a diffusing particle at site $i$ at time $t$; it is given by

$$
\Delta^{\prime} P_{i}(t)=\frac{\partial}{\partial t} P_{i}(t)
$$

where the quasi-Laplacian $\Delta^{\prime}$ is defined as

$$
\Delta^{\prime} P_{i}=\sum_{j}\left(P_{j}-P_{i}\right),
$$

with $j$ running over the nearest neighbours of site $i$ in the fractal structure. This equation is usually studied with the initial condition

$$
P_{i}(0)=\delta_{i, 0}
$$

Equation (1) can formally be solved in terms of the eigenfunctions of the $\Delta^{\prime}$ operator, obtained (e.g. in an obvious continuous description) from

$$
\left(\Delta^{\prime}+E\right) \Psi_{E}(\mathbf{r})=0 .
$$

(The presence of some suitable boundary conditions has to be assumed.) The solution to the diffusion equation can then be written as

$$
P(\mathbf{r}, t)=\int d E \rho(E) \Psi_{E}^{*}(0) \Psi_{E}(\mathbf{r}) \mathrm{e}^{-E t}
$$

$\rho(E)$ represents the spectral density of the $\Delta^{\prime}$-operator. This is closely related to the vibrational spectrum of the fractal, since the vibrations have in a similar way to obey the equation ${ }^{3}$

$$
\Delta^{\prime} u(\mathbf{r}, t)=\frac{\partial^{2}}{\partial t^{2}} u(\mathbf{r}, t)
$$

The vibrational modes are described by the same functions $\Psi_{E}(\mathbf{r})$ and they correspond to frequencies $\omega=\sqrt{E}$. Since the low frequency spectral density $\tilde{\rho}(\omega)$ of phonons behaves on a regular $d$-dimensional lattice like $\omega^{d-1}$, the spectral or fracton dimension $\tilde{d}$ was defined [3] through the relation

$$
\tilde{\rho}(\omega) \sim \omega^{\tilde{d}-1} \quad(\omega \rightarrow 0)
$$

which is equivalent to

$$
\rho(E) \sim E^{-1+\tilde{d} / 2} \quad(E \rightarrow 0) .
$$

${ }^{3}$ The equation actually describes a rather peculiar form of scalar vibrations. 
Using this behaviour in (5) shows us immediately that the solution to our diffusion equation has the asymptotic property

$$
P(0, t) \sim t^{-\tilde{d} / 2} \quad(t \rightarrow \infty) .
$$

The diffusion of a particle on a fractal structure is usually described in terms of the effective dimensionality $d_{w}$ of the random walk problem, through the relation

$$
t \sim R^{d_{w}} \quad \text { with } \quad R^{2}=\left\langle r^{2}(t)\right\rangle,
$$

which links the mean square displacement to the diffusion time. Alternatively, one can define an exponent $\nu$ with

$$
\left\langle r^{2}(t)\right\rangle \sim t^{2 \nu} \quad\left(\nu=1 / d_{\mathrm{w}}\right) .
$$

The exponent $d_{\mathrm{w}}$ is not independent from the spectral dimension $\tilde{d}$; it can be argued that the diffusion probability should behave for large times as

$$
P(\mathbf{r}, t) \approx r^{-D} f\left(r^{d_{w}} / t\right)
$$

where the factor $r^{-D}$ appears for reasons of normalization. With

$$
g(x)=x^{-D / d_{w}} f(x)
$$

this can also be written as

$$
P(\mathbf{r}, t) \approx t^{-D / d_{w}} g\left(r^{d_{w}} / t\right)
$$

Comparison with (9) then tells us that

$$
\tilde{d}=\frac{2 D}{d_{\mathrm{w}}} .
$$

This equation allows us to determine the low frequency scaling of the phonon spectral density through the asymptotic behaviour of the diffusion for $t \rightarrow \infty$.

It was soon discovered [5] that the previously defined exponents are also related to the resistivity exponent $\zeta$, defined through

$$
\Omega(r) \sim r^{\zeta} \quad(r \rightarrow \infty)
$$

where $\Omega(r)$ is the electrical resistance between two points at a distance $r$ on the fractal, if the bonds of the fractal structure are replaced by identical resistances. On a regular $d$-dimensional lattice we can estimate $\Omega(r)$ from the picture that between two points $\mathrm{A}$ and $\mathrm{B}$ at a distance $r$ there exist roughly $r^{d-1}$ parallel conducting paths of length (and thus of resistivity) $r$. This leads to the formula

$$
\Omega(r) \sim \frac{1}{\sigma} \frac{r}{r^{d-1}}=\frac{1}{\sigma} r^{2-d} .
$$


$\sigma$ is the local conductivity, and this should contain the information about the fractal structure. This quantity can be calculated from the Einstein relation as a function of the density $n$ and the diffusion coefficient $C$ :

$$
\sigma=\frac{e^{2}}{k_{\mathrm{B}} T} n C .
$$

The diffusion coefficient at a distance $r$ on the fractal can be estimated as follows:

$$
C=\frac{d}{d t}\left\langle r^{2}(t)\right\rangle \sim t^{2 / d_{w}-1} \sim r^{2-d_{w}} .
$$

The density $n$ obviously behaves like

$$
n \sim r^{D-d}
$$

and thus

$$
\sigma \sim r^{D-d_{w}+2-d}
$$

Using this result in (16) gives us

$$
\zeta=d_{\mathrm{w}}-D .
$$

This relation, which can also be derived from the Green function of the $\Delta^{\prime}$ operator [6], provides us with an independent way of determining the dynamical exponents through a direct calculation of the exponent $\zeta$.

\section{Diffusion on Two-dimensional Random Walks}

The diffusion problem on random two-dimensional substrates generated by random walks has led in the literature to some contradictory statements. It has been claimed [7] that in this case, since $D=d=2$, the random walk is homogeneous in space, and we should therefore have $\zeta=0$, as in the regular two-dimensional lattice. This would lead to $d_{\mathrm{w}}=2$ or $\nu=1 / 2$ and hence $\tilde{d}=2$. Resistivity calculations [8], on the other hand, seemed to favour $\zeta=1$, hence $d_{\mathrm{w}}=3$ and $\tilde{d}=4 / 3$. This has led the present authors to an extensive numerical study of the diffusion of a particle during 100 time steps on random walks of 20,000 steps on the square lattice [9], which strongly supported the results of the resistivity calculations with $\nu=1 / 3$. These results were contradicted by a calculation [10] of the diffusion on $N$-step random walks in the limit $N \rightarrow \infty$, which led to the result

$$
\nu-\frac{1}{2} \sim 1 / \ln N \text {. }
$$

In order to understand what is going on, one should remember that the infinite two-dimensional random walk is known to visit all sites of the lattice and diffusion on a regular lattice is always described by $\nu=1 / 2$. The asymptotic $t \rightarrow \infty$ behaviour of the diffusion on a finite random walk, on the other hand, can only be described by $\nu=0$. This means that all finite, but eventually large, random walks can be described by an effective $\nu_{\text {eff }}(t)$, for which we should 
thus expect that it varies from $1 / 2$ for $t=0$ to 0 for $t \rightarrow \infty$. We believe that the dynamical behaviour of a large two-dimensional random walk can be characterized by three different regimes (either in time or in diffusion length): an initial regime, where the normal two-dimensional character is manifested (e.g. $\nu=1 / 2)$, an intermediate regime where the true fractal behaviour of the walk manifests itself, and a final regime where the limiting boundary of the walk starts hindering further diffusion. In this sense, the random walk situation could have some resemblance with that of realistic systems like Silica aerogels[11], where the fractal behaviour is found for lengths $L$ satisfying $a \ll L \ll \xi$, where $a$ is an atomic length scale and $\xi$ some correlation length. In our case, $a$ would rather be the average size of densely filled clusters and instead of $\xi$ we should have the average diameter of the random walk.

It is illuminating to compare the finite random walk with, e.g., finite realisations of Sierpinsky gaskets. We calculated exactly $\left\langle r^{2}(t)\right\rangle$ on respectively the 4-th, 5-th, 6-th and 7-th generation of simple Sierpinsky triangular gaskets for times up to $t=1200$, starting from an arbitrary interior point. Whereas the infinite gasket corresponds to $\nu=\ln 2 / \ln 5 \approx 0.43$, our numerical study for the finite gaskets showed an effective $\nu$-exponent fluctuating around the value 0.43 up to a crossover time $t_{x}$, which scales with the size $R$ of the system like $t_{x} \sim R^{1 / \nu}$.

The situation is quite different in the random walk case. The diameter of an $N$-step walk is proportional to $\sqrt{N}$; if the random walk would be comparable to a finite-size fractal with diffusion exponent $\nu=1 / 2$, we should expect to see a rather flat $\nu_{\text {eff }}(t)=1 / 2$ up to $t \approx N$. We always see, however, that $\nu$ drops rather sharply to a much lower value on a much shorter time scale. The actual value seems to saturate around $1 / 3$ with a rather long flat plateau, although a simple $\log -\log$ analysis is too crude to give an accurate estimate (an alternative estimate was given elsewhere[9]). If this is indeed the correct value for $\nu$ in the fractal regime, as we suppose, we should see the crossover to $\nu=0$ appear only at times of the order $t_{x} \approx N^{3 / 2}$. For $N=2,000$, e.g., this already means $t_{x} \approx 100,000$, which is unfortunately too high to study numerically.

In conclusion, we want to stress that the results obtained by Manna et al.[10] are only relevant for the initial time regime. Indeed, their 100-step blind ant walks correspond perhaps to 60 -step myopic ant walks, as studied by us; these short diffusion times are implemented on very long random walks, up to 640,000 steps in their case, to be compared with our 20,000 steps. It is clear that they are in a completely different regime for the $t / N$-ratio, i.e. they are testing compact regions of the random walk structure. Furthermore, we have extended our Monte Carlo diffusions on a 20,000-step random walk up to $t=10,000$, confirming in a crude log-log analysis the picture of a long flat plateau around $1 / 3$ for the $\nu$-values.

In order to obtain an independent confirmation of our result, we have also investigated $P(0, t)$, the probability for return to the origin during a diffusion process, whose behaviour is described by (9). We have therefore generated large samples of random walks of varying lengths, and we solved exactly the diffusion 
equation on their substrates for the occupation probabilities $P(\underset{\sim}{\mathbf{r}}, t)$ for $t \leq 2000$. Through log-log calculations we obtain the following results: $\tilde{d}=1.08$ for $N=$ 5,000 and $d=1.18$ for $N=20,000$ (sample size: 1380). A linear extrapolation in $1 / N$ yields $\tilde{d}=1.22$, whereas an extrapolation in $1 / \ln N$, in analogy with the calculations of Ref. [10], gives $\tilde{d}=1.30$. These results are compatible with $\tilde{d}=4 / 3\left(\right.$ or $\left.d_{\mathrm{w}}=3\right)$ and they rather clearly exclude the value $\tilde{d}=2\left(\right.$ or $\left.d_{\mathrm{w}}=2\right)$.

\section{Hierarchical Model with Random Walk Similarities}

In order to understand better the results from our simulations, we tried to construct a deterministic model of which one could hope that it possesses the essential properties that lead to the anomalous dynamical behaviour as was observed in the two-dimensional random walks. We therefore looked at the patterns that were formed by some large, but finite, random walks on a regular lattice. These patterns seem to be composed of many densely visited regions of various sizes, interconnected by narrow pathways. This scheme, moreover, seems to repeat itself over many length scales. Led by these considerations, we have constructed in a hierarchical way a fractal structure that incorporates the same succession of dense regions and narrow links, hoping to be able to calculate exactly some dynamical properties, like diffusion rate or resistivity, on these fractals.

In Fig. 1 we present the iterative construction of such a model, in which at each step four blocks of the previous step are connected with one another into a larger block. Since there is still some variety in the way in which one performs these connections, we actually have a class of models.

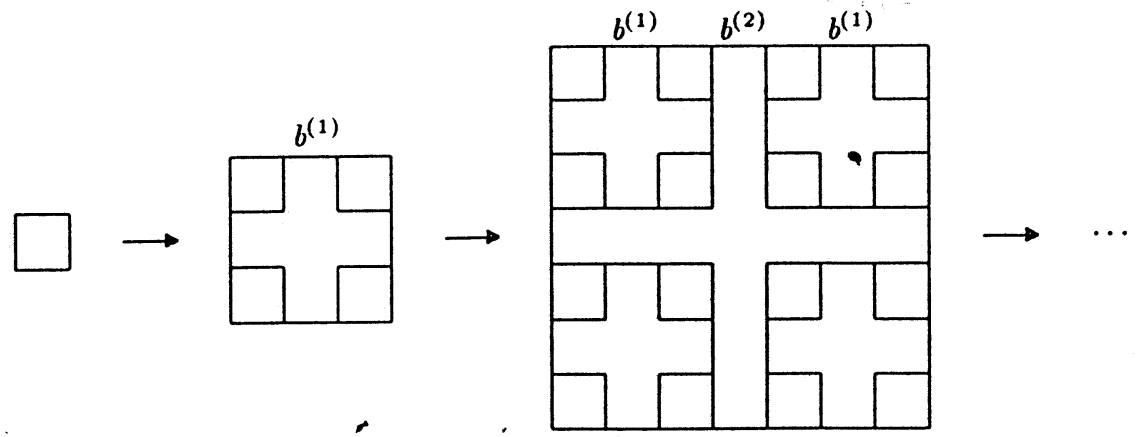

Fig. 1. Iterative construction of the class of fractal models, depending on a family of parameters $\left\{b^{(n)}\right\}$.

The links on the structure represent paths along which a particle may diffuse. The elementary units of the zeroth step of the structure are squares with some 
initial transition rate $a$ along the sides and zero transition rate along the diagonal. The links connecting these elementary units may be classified according to the order in which they appear in the iterative construction of the fractal. We may thus introduce transition rates $b^{(n)}$ with $n=1,2,3, \ldots$ for this class of links. The most simple choice might seem to take $b^{(n)}=b=a$ for all $n$, which would correspond to the problem of a regular lattice with missing links. Keeping in mind the pictures of our random walk samples, however, we want to leave open the possibility to have longer links between the blocks at higher steps of the construction. The essential property of these links should be that they have a smaller transition rate than the bonds of the elementary squares.

We have solved the diffusion problem on this class of models, characterized by the transition rates $a$ and $b^{(n)}$, through a renormalization procedure by decimation. By this procedure a block, constructed from four unit squares linked together, is mapped onto a single square (see Fig. 2), by decimating out 12 from the original 16 sites of the block. By repeating this decimation $n$ times, the linear size of the blocks is reduced after the $n$-th step from $\left(2^{n}-1\right)$ units to $\left(2^{n-1}-1\right)$ units, which means that asymptotically the lengths are rescaled by a factor 2 .
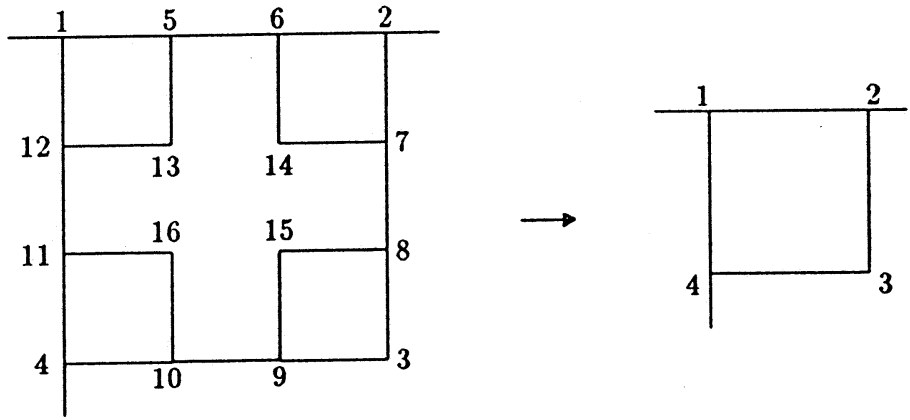

Fig. 2. Scheme for the renormalizatio procedure by decimation for the diffusion equations.

We have numbered the sites of our original block from 1 to 16 in such a way that sites numbered from 1 to 4 are saved by the decimation procedure (see Fig. 2). We suppose that the transition rates for the site occupation probabilities inside this block are given by $a, b$ and $c$ (See Fig. 3), where $a$ and $b$ are regular nearest neighbour transition rates (the $b$ being equal to $b^{(1)}$ in the first step), and $c$ is a diagonal transition rate. On the initial fractal we suppose $c=0$, but since it acquires a nonzero value through the renormalization procedure, we include it in our calculation. Furthermore, sites 1 through 4 may or may not have an outgoing link with a transition rate $b^{(n)}$ (with $n>1$ ), depending on their position in the fractal. We indicate this transition rate by $b_{1}$, which must 
be zero if the link is missing.

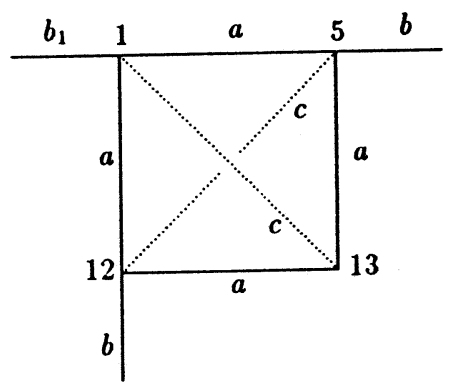

Fig. 3. Transition rates for the diffusion.

Let $P_{k}(t)$ indicate the probability for finding the particle at site $k$ at time $t$. These quantities obey the master equation

$$
\frac{d P_{k}}{d t}=\sum_{j} W_{k j}\left(P_{j}-P_{k}\right),
$$

where $W_{i j}=W_{j i}$ denote the already mentioned transition rates. We define $X_{k}(\lambda)$ as the Laplace transform of the $P_{k}(t)$. From $(3)$ these $X_{k}(\lambda)$ obey a set of 16 equations, which may easily be constructed from Fig. 2. Using the definition

$$
p=2 a+c,
$$

and denoting with $X_{k}^{0}(\lambda)$ the Laplace transform of the occupation probability of an external neighbour to site $k(k=1, \ldots, 4)$, we have, e.g.,

$$
\begin{aligned}
\left(\lambda+p+b_{1}\right) X_{1}= & a\left(X_{5}+X_{12}\right)+c X_{13}+b_{1} X_{1}^{0} \\
\cdots & \cdots \\
\left(\lambda+p+b_{1}\right) X_{4}= & a\left(X_{10}+X_{11}\right)+c X_{16}+b_{1} X_{4}^{0} \\
(\lambda+p+b) X_{5}= & a\left(X_{1}+X_{13}\right)+c X_{12}+b X_{6} \\
\cdots & \cdots \\
(\lambda+p+b) X_{12}= & a\left(X_{1}+X_{13}\right)+c X_{5}+b X_{11} \\
(\lambda+p) X_{13}= & a\left(X_{5}+X_{12}\right)+c X_{1} \\
\cdots & \cdots \\
(\lambda+p) X_{16}= & a\left(X_{10}+X_{11}\right)+c X_{4}
\end{aligned}
$$

A straightforward elimination procedure of the functions $X_{5}$ through $X_{16}$ can always be normalized in such a way that we end up with the original value of $a$ in equations such as

$$
\left(\lambda^{\prime}+p^{\prime}+b_{1}^{\prime}\right) X_{1}=a\left(X_{2}+X_{4}\right)+c^{\prime} X_{3}+b_{1}^{\prime} X_{1}^{0}
$$


and analogous equations for $X_{2}, X_{3}$ and $X_{4}$. We defined

$$
p^{\prime}=2 a+c^{\prime} \text {. }
$$

Since $a$ remains constant, we may arbitrarily choose it to be equal to unity from now on: $a=1$.

Since we want to study the long time behaviour of these equations, we can restrict ourselves to small values of $\lambda$, and hence we expand the obtained equations in ascending powers of $\lambda$. This leads to

$$
\begin{aligned}
b^{(n) \prime} & =\gamma b^{(n+1)}, \\
c^{\prime} & =\frac{b(1+c)}{2(1+c)+b(3+c)}+\mathcal{O}(\lambda), \\
\lambda^{\prime} & =4 \gamma \lambda, \\
\gamma & =\frac{(1+c)+b(2+c)}{b(1+c)}+\mathcal{O}(\lambda) .
\end{aligned}
$$

One should remark that, together with their transformation, the b's shift down one step in their eventual hierarchy of the $\left\{b^{(n)}\right\}$-values.

It is immediately clear from (33) that, if initially all $b^{(n)}$ are equal, they will finally be renormalized to infinity, since $\gamma>1$. In this case, we obtain a fixed point

$$
\begin{aligned}
& b^{\star}=\infty \\
& c^{\star}=\sqrt{2}-1
\end{aligned}
$$

and from (35) follows in this case

$$
\left(\frac{\lambda^{\prime}}{\lambda}\right)^{\star}=4+2 \sqrt{2} .
$$

Since each step in the decimation procedure corresponds to a length rescaling by a factor 2 , we expect from (10) that this implies a rescaling of the time by a factor $2^{d_{w}}$, from which we may infer that

$$
d_{w}=\frac{\ln \left(\lambda^{\prime} / \lambda\right)}{\ln 2} .
$$

Our infinite- $b$ fixed point corresponds then to $d_{\mathrm{w}}=2.77$ or $\tilde{d}=1.44$.

Another possibility is to start from a model, in which the $b^{(n)}$ are not equal to one another, but become smaller with growing $n$, indicating in this way some lengthening of the effective diffusion path along these links. If the hierarchy of $b^{(n)}$-values is chosen properly, one might obtain any desired finite fixed point value for $b^{\star}$. In such cases we have:

$$
\begin{aligned}
c^{\star} & =\frac{\left[2\left(b^{\star}\right)^{2}+4 b^{\star}+1\right]^{1 / 2}-b^{\star}-1}{b^{\star}+2}, \\
\left(\frac{\lambda^{\prime}}{\lambda}\right)^{\star} & =\frac{2\left(c^{\star}+1\right)}{c^{\star}} .
\end{aligned}
$$


In order to obtain these values we should, at least asymptotically for large $n$, have started with a situation where

$$
b^{(n)} \simeq b f^{-n}
$$

with

$$
f=\left(c^{\star}+1\right) / 2 c^{\star} .
$$

From these formulas, it is clear that, when $b^{\star}$ goes from 0 to $\infty, c^{\star}$ increases monotonically from 0 to $\sqrt{2}-1$ and $f$ from $\infty$ to $1+\sqrt{2} / 2$.

If in (43) $f$ is smaller than $f_{c}=1+\sqrt{2} / 2$, then $b$ always iterates to $b^{\star}=\infty$ and $c$ to $c^{\star}=\sqrt{2}-1$.

Since our renormalization steps correspond to a rescaling of the lengths by a factor 2, we have in general (see Fig. 4)

$$
d_{w}= \begin{cases}2+\ln f / \ln 2 & \left(f \geq f_{c}\right) \\ 2+\ln f_{c} / \ln 2 & \left(f<f_{c}\right) .\end{cases}
$$

This means that we have a whole class of models with nonuniversal diffusion dimensions $2.77 \leq d_{w} \leq \infty$. One special case immediately draws our attention. The $b^{(n)}$ are diffusion strengths on links between clusters with a linear dimension $2^{n}$. In realistic fractal structures grown by some random procedures, a good guess would be that links between such clusters would themselves have lengths of the order $2^{n}$. This can be modeled in our scheme by making the diffusion strengths $b^{(n)}$ shrink by factors $f=2$. This special and obvious choice leads to the values $b^{\star}=4, c^{\star}=1 / 3,\left(\lambda^{\prime} / \lambda\right)^{\star}=8, d_{\mathrm{w}}=3$, and it gives us the Alexander-Orbach value $\tilde{d}=4 / 3[3]$.

Completely independent of the previous renormalization calculation, we have also performed [12] an iterative calculation of the electrical resistivity between opposing diagonal points of the fractal structure in increasing order of the construction. For all $f$, these resistivities $\Omega_{n}$ scale like $2^{n \zeta}$, where $\zeta=d_{\mathrm{w}}-2$ with $d_{\mathrm{w}}$ exactly given by (45). This calculation thus proves that relation (21) is valid for our whole class of models.

\section{A Model with Logarithmic Time Dependence}

It has been known for many years that some special models may give rise to extremely slow diffusion, such that (5) has to be replaced with a logarithmic time dependence

$$
R \sim(\ln t)^{k}
$$

for some value of $k$. Such behaviour has been demonstrated for a particle on a one-dimensional chain, subject at each site to an independent random bias [13]. Another mechanism leading to the same behaviour is that arising in a comb-like structure with teeth of variable length, along which a bias field pushes the particle towards the tip. A suitable power law distribution of teeth lengths can be shown to imply delay times, which make diffusion along the comb basis logarithmic [14]. There is some numerical evidence $[15,16]$ that the same combination 


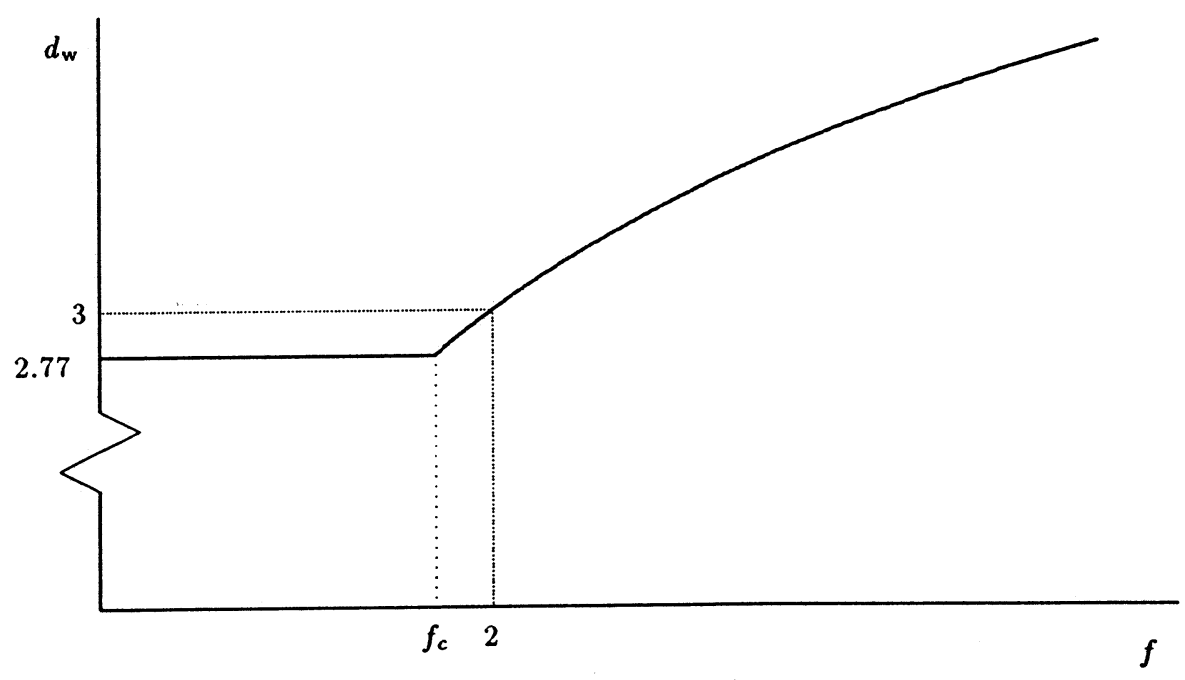

Fig. 4. Exponent $d_{w}$ versus rescaling factor $f$ for the diffusion family $\left\{b^{(n)}\right\}$.

of dangling ends and a bias field can give rise to a logarithmic behaviour also in higher-dimensional models like the infinite incipient cluster of percolation. This was recently confirmed by a renormalization group (RG) calculation on the hierarchical $\mathrm{T}$-fractal [17], and we will briefly report on these results. The calculation is of some methodological interest, since the $\mathrm{RG}$ recursion equations are singular at the fixed point, such that the standard analysis for extracting the asymptotic behaviour has to be reconsidered.

The iterative construction of the T-fractal, which has a central site $j=0$, is illustrated in Fig. 5. It is a ramified structure with fractal dimension $D=\ln 3 / \ln 2$. The resulting structure, after two iterations, is shown in Fig. 6.

A diffusing particle hops between nearest neighbour sites of the T-structure according to the master equation, equivalent to (1):

$$
P_{i}(t+\tau)=P_{i}(t)+\sum_{i \neq j}\left[W_{i j} P_{j}(t)-W_{j i} P_{i}(t)\right],
$$

where the $W_{i j}$ represent the hopping probabilities during time $\tau$. It is assumed 


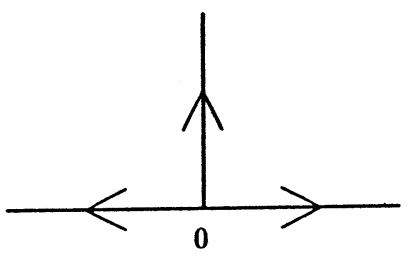

(a)

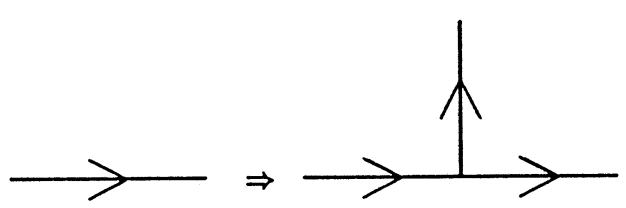

(b)

Fig. 5. (a) Starting configuration and (b) iterative step for the construction of the T-fractal.

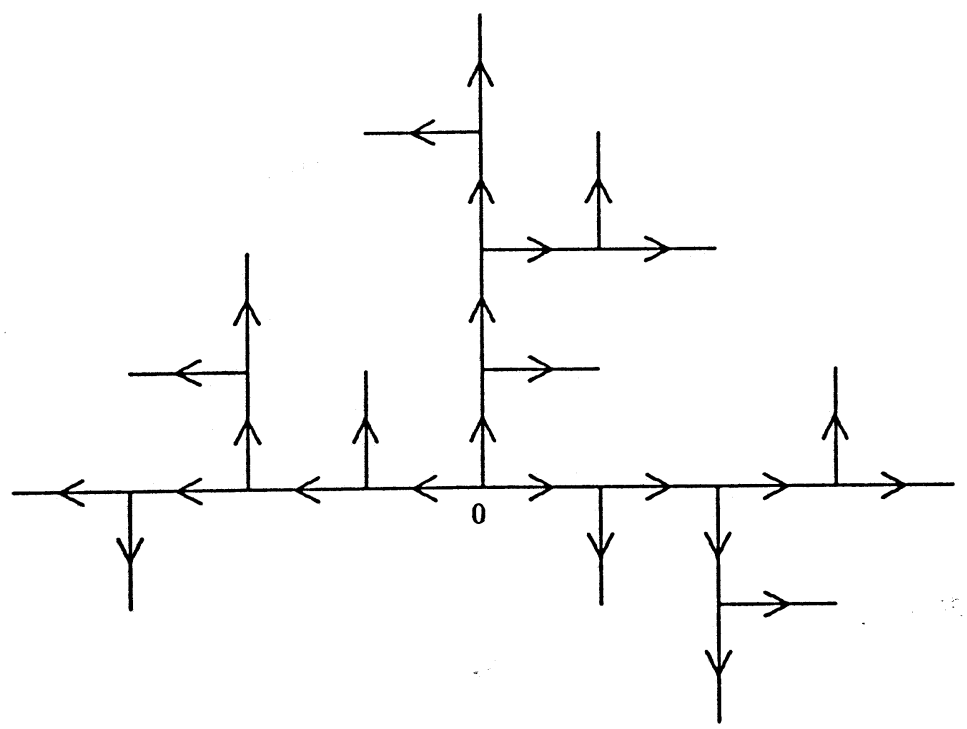

Fig. 6. T-fractal after two steps in the iterative construction.

that $W_{i j}=W_{+}$or $W_{-}$, according to whether one goes from $j$ to $i$ following the direction of the bond arrows of Fig. 4 or against them. In order to preserve positive probabilities, the following relations should be satisfied:

$$
\begin{array}{r}
2 W_{+}+W_{-} \leq 1 \\
3 W_{+} \leq 1 .
\end{array}
$$

We will furthermore assume that

$$
W=\frac{W_{-}}{W_{+}} \leq 1,
$$


which means, as a consequence of the direction of the arrows, that there is a bias, pushing the particle to higher "chemical" distance from the origin. We put $\tau=1$, and introduce discrete Laplace transforms [18]:

$$
\tilde{P}_{i}^{0}(z)=\sum_{n=0}^{\infty} P_{i}(n)(1+z)^{-1-n}
$$

This transforms (47) into

$$
(1+z) \tilde{P}_{i}^{0}(z)-P_{i}(0)=\tilde{P}_{i}^{0}(z)+\sum_{j \neq i}\left[W_{i j} \tilde{P}_{j}^{0}(z)-W_{j i} \tilde{P}_{i}^{0}(z)\right] .
$$

With the definitions $\omega=z / W_{+}$and $\tilde{P}_{k}(\omega)=W_{+} \tilde{P}_{k}^{0}(z)$, and using the initial condition $P_{i}(0)=\delta_{i, 0}$, this can also be written as

$$
\left(\alpha(i) \omega+\sum_{j \neq i} \frac{W_{j i}}{W_{+}}\right) \tilde{P}_{i}(\omega)=\sum_{j \neq i} \frac{W_{i j}}{W_{+}} \tilde{P}_{j}(\omega)+\delta_{i, 0}
$$

Equation (50) actually gives us $\alpha(i)=1$ for all $i$, but since the RG procedure will generate three different values $\alpha_{0}, \alpha_{1}$ or $\alpha_{3}$, according to whether $i$ is the origin or a site with coordination number 1 or 3 , we introduce from the beginning such a variable $\alpha(i)$. The only parameters entering (51) will thus be $\alpha=\left(\alpha_{0}, \alpha_{1}, \alpha_{3}\right)$ and $W$.

The RG transformation is now performed by eliminating from the equations (51) the $\tilde{P}_{j}$ corresponding to sites $j$ introduced at the last step in the iterative construction of the T-fractal. This corresponds to performing the inverse of the operation illustrated in Fig. 5(b). The sites of the old structure which are not decimated have distances reduced by a factor 2 , if measured in terms of the new lattice spacing. The decimated equations can be expressed in a form similar to that of the original equations, if we use the following renormalized parameters:

$$
\begin{aligned}
W^{\prime} & =W^{2} \\
\alpha_{0}^{\prime} \omega^{\prime} & =\left(\alpha_{0} \omega+3\right)\left(\alpha_{3} \omega+2+W-\frac{W}{\alpha_{1} \omega+W}\right)-3 W-3 \\
\alpha_{1}^{\prime} \omega^{\prime} & =\left(\alpha_{1} \omega+W\right)\left(\alpha_{3} \omega+2+W\right)-2 W-W^{2} \\
\alpha_{3}^{\prime} \omega^{\prime} & =\left(\alpha_{3} \omega+2+W\right)\left(\alpha_{3} \omega+2+W-\frac{W}{\alpha_{1} \omega+W}\right)-2-3 W-W^{2} \\
\tilde{P}_{i}^{\prime} & =\frac{\alpha_{1} \omega+W}{\left(\alpha_{1} \omega+W\right)\left(\alpha_{3} \omega+2+W\right)-W} \tilde{P}_{i} .
\end{aligned}
$$

In order to see how the further analysis should be performed, we first consider the unbiased case, corresponding to the (unstable!) fixed point $W^{*}=1$ of (52). 
Large $t$-values correspond to small $\omega$, and in the limit $\omega \rightarrow 0$ and $W=1(53-55)$ become:

$$
\begin{aligned}
& \alpha_{0}^{\prime} \omega^{\prime}=\left(2 \alpha_{0}+3 \alpha_{1}+3 \alpha_{3}\right) \omega \\
& \alpha_{1}^{\prime} \omega^{\prime}=\left(3 \alpha_{1}+\alpha_{3}\right) \omega \\
& \alpha_{3}^{\prime} \omega^{\prime}=\left(3 \alpha_{1}+5 \alpha_{3}\right) \omega .
\end{aligned}
$$

Under repeated action of such a transformation, the value of $\omega^{\prime} / \omega$ and the vector $\alpha$ will tend respectively to the largest eigenvalue and the corresponding eigenvector of the matrix

$$
\left(\begin{array}{lll}
2 & 3 & 3 \\
0 & 3 & 1 \\
0 & 3 & 5
\end{array}\right) .
$$

Since this largest eigenvalue is 6 , and since $\omega$ plays here the same role as the Laplace transform variable $\lambda$ introduced for the study of the model (23) in the previous section, we can now write in analogy with (40):

$$
d_{\mathrm{w}}=\frac{\ln \left(\omega^{\prime} / \omega\right)^{*}}{\ln 2}=\frac{\ln 6}{\ln 2},
$$

which describes indeed the unbiased diffusion on the T-fractal [18].

If we try to use the same method in the neighbourhood of the second fixed point $W^{*}=0$, which controls the biased diffusion, we get into trouble since the transformation matrix for $\alpha$ now becomes to leading order in $W$ :

$$
\boldsymbol{\alpha}^{\prime} \omega^{\prime}=\left(\begin{array}{ccc}
1 & 3 / W & 3 \\
0 & 2 & W \\
0 & 2 / W & 3
\end{array}\right) \boldsymbol{\alpha} \omega .
$$

Although there is a regular largest eigenvalue 4 , we can not repeat the previous analysis since we will never reach a finite $\boldsymbol{\alpha}^{*}$ : under the renormalization the $\alpha_{0}$ and $\alpha_{3}$ will for small $W$ at every step be multiplied with larger and larger factors $\left(W^{2^{n}}\right)^{-1}$. After $n$ iterations, the leading contribution to $(\alpha \omega)^{(n)}$ can easily be estimated from (62) to be of the form

$$
(\alpha \omega)^{(n)} \approx\left(a_{0} 2^{n} / W^{2^{n}}, a_{1} 2^{n}, a_{3} 2^{n} / W^{2^{n}}\right)
$$

the $a_{i}$ 's being suitable constants depending on initial conditions. This means that, if one wants to retain the original form (51) for the diffusion with a fixed point value for the vector $\alpha$, one should renormalize the variable $\omega^{(n)}$ not by multiplying it with a constant factor at each step as $\Lambda^{n} \omega$, but in the following form:

$$
\omega^{(n)}=\omega 2^{n} / W^{2^{n}}
$$

or

$$
\ln \omega=\ln \left(\omega^{(n)} 2^{-n}\right)+2^{n} \ln W .
$$


Simultaneously, the average distance travelled by a diffusing particle renormalizes as

$$
\left\langle R^{(n)}\right\rangle \approx 2^{-n}\langle R\rangle
$$

or

$$
\langle R\rangle \sim 2^{n} \sim \frac{|\ln \omega|}{|\ln W|} \sim \frac{\ln t}{|\ln W|} .
$$

(Remark that the main contribution to the Laplace transform comes from the region of values where $\omega t \approx 1$.) For a more detailed derivation of this result, we refer to Reference [17].

As anticipated, the singular character of the $R G$ mapping gives rise to logarithmic diffusion. An extra result is the logarithmic dependence on $W$, which has been conjectured on the basis of numerical evidence or on heuristic arguments $[16]$.

\section{Conclusions}

As anticipated in the introduction, the applications of the dynamical RG discussed here have shown how far reaching this method can be for the qualitative understanding of very complicated, and sometimes controversial, mechanisms of diffusion on fractal structures. The key to success is of course an appropriate choice of the hierarchical model on which the RG should be applied. Even if the relevance of such results to the corresponding problems on real random fractal structures can always be questioned, there is no doubt that the exercise is suggestive and instructive on its own. For the diffusion phenomena discussed here, more realistic models can only be treated by numerical methods, and even then with much difficulty. Certainly the analytical insight provided by our toy models is of help to our general understanding, by providing simplified but clear mechanisms of explanation. In addition, considering borderline applications can lead to interesting methodological spinoffs for the RG strategy itself. The relevant example here is our singular RG recursion for logarithmic diffusion. A singular transformation, at first sight, seems to contrast with the common wisdom about RG strategies. Here we showed that such singular transformation can be handled within an exact treatment and is the natural mechanism for explaining the phenomenon.

\section{References}

1. B.B.Mandelbrot, The Fractal Geometry of Nature (Freeman, San Francisco, 1982).

2. P.G. de Gennes, Scaling Concepts in Polymer Physics (Cornell University Press, Ithaca, 1979).

3. S.Alexander and R.Orbach, J.Phys. (Paris) Lett. 43, L 625 (1982).

4. S.Alexander, J.Bernasconi, W.R.Schneider and R.Orbach, Rev.Mod. Phys. 53, 175 (1981).

5. Y.Gefen, A.Aharony and S.Alexander, Phys.Rev.Lett. 50, 77 (1983). 
6. M.E.Cates, J.Phys. (Paris) 46, 1059 (1985).

7. S.Havlin, G.H.Weiss, D.Ben-Avraham and D.Movshovitz, J.Phys. A 17, L849 (1984).

8. J.R.Banavar, A.B.Harris and J.Koplik, Phys.Rev.Lett. 51, 1115 (1983).

9. R.Dekeyser, A.Maritan, and A.L.Stella, Phys.Rev.Lett. 58, 1758 (1987) and Phys.Rev. A 36, 2338 (1987).

10. S.S.Manna, A.J.Guttmann and B.D.Hughes, Phys.Rev. A 39, 4337 (1989).

11. D.W.Schaefer and K.D.Keefer, Phys.Rev.Lett. 56, 2199 (1986).

12. R.Dekeyser, A.Maritan and A.Stella, Phys.Rev. A 40, 5299 (1989).

13. Y.G.Sinai, Theory of Prob. and its Appl. 27, 256 (1982).

14. S.Havlin, A.Bunde, Y.Glaser and H.E.Stanley, Phys.Rev. A 34, 3492 (1986).

15. D.Stauffer, J.Phys. A 18, 1827 (1985).

16. A.Bunde, H.Harder, S.Havlin and H.E.Stanley, J.Phys. A 20, L865 (1987).

17. A.Maritan, G.Sartoni and A.L.Stella, Phys.Rev.Lett. 71, 1027 (1993).

18. A.Giacometti, A.Maritan and A.L.Stella, Int.J.Mod.Phys. B 5, 709 (1991).

This article was processed using the $\mathrm{LAT}_{\mathrm{E}} \mathrm{X}$ macro package with LMAMULT style 